\title{
El crecimiento económico en El Salvador de 1950 a 1996
}

$\mathrm{D}$ espués de más de una década de desgaste económico y social por la guerra civil en El Salvador, en los años novenla se ha revivido el interés por el crecimiento económico, que se mide normalmente por la tasa de crecimiento del produclo intemo bruto (PIB) en términos reales. Este interés se ve reforzado por un repunte en el crecimiento económico en los primeros años de los noventa, posteriores a la firma de los acuerdos de paz de Chapultepec, y por la caída en la tasa de crecimiento económico en 1996 que ha dejado a muchos preocupados por el desempeño de la economía en ese año'.

No cabe duda que para muchos expertos hay una claridad del significado de la tasa de crecimiento económico, pero me pregunto si en el debate político nacional, en los informes de prensa y para el público salvadoreño, en general, existe una idea adecuada de las dimensiones del crecimiento económico y de su significado. El propósito de este pequeño trabajo es poner al alcance de la mayoría de personas una noción de cómo interpretar las cifras del crecimiento económico y de esta manera proporcionar un instrumento para interpretar claramente su significado. No es mi propósito explicar cuáles son las causas del crecimiento, ni de dónde provienen, si no más bien explicar qué significa crecer a una determinada tasa $x$ ó $y$, a cuánto hemos crecido en las últimas décadas, y qué significado tiene esto para la formación de expectativas en cuanto al futuro del bieneslar material en El Salvador.

\section{1. ¿Cómo ha evolucionado la producción?}

En esta sección presento brevemente cómo ha evolucionado la producción medida a través del producto intemo bruto (PIB) y del producto interno bruto per cápita (PIBP), ambas variables medidas en términos reales, es decir, que se han hecho los ajustes necesarios para eliminar los efectos nominales que tienen sobre su medición los cambios en el nivel general de precios. En el apéndice 
número uno se puede encontrar una tabla de los datos utilizados y sus respectivas fuentes.

La selección de eslas dos variables, PIB y PIBP, como indicadores del comportamiento económico se hace en parte por convención y en parte por conveniencia, pues ambas series se pueden conseguir con relativa facilidad, y para ser unas variables tan generales su estimación también es bastante precisa. Principalmente, el PIBP es una buena aproximación como medida del potencial de bienestar material de un país, pues lo que nos está dando es la producción tolal por habilante durante un año determinado. Sin embargo, no hay que olvidar que éstas sólo nos dan una aproximación, pues no estamos tomando en cuenta otros factores del bienestar económico y social de un país, como lo son la distribución del ingreso, la esperanza de vida, la mortalidad infantil, elc. Esta advertencia es un primer paso para enlender lo que se dice cuando se habla de crecimiento económico, pues para entender hay que tener claridad de lo que se dice y de lo que no se dice.

Teniendo en cuenta la aclaración anterior, veamos el comportamiento del PIB en los años 1950-1996. En la Gráfica 1 se presenta la evolución del PIB en relación con los precios de 1990 . Vemos que aumenta sin reparos desde 1950 hasta 1978, donde se alcanza un pico en la producción, luego viene la caída de los primeros cuatro años de la guerra civil, y el comportamiento positivo comienza nuevamente en 1983. Es de notar que salvo en los primeros cuatro años del conflicto armado, en todos los años ha habido un crecimiento positivo del PIB, aunque éste se ha dado a diferentes ritmos cada año. Esto ya nos dice algo sobre nuestra economía, y es que es capaz de lograr un crecimiento positivo en la producción total en casi todos los años. Este aumento en la producción se debe principalmente al aumento de los recursos productivos y su utilización, principalmente del acervo de capital en la economía, de las horas de trabajo que dependen del número de trabajadores y de las innovaciones tecnológicas que principalmente provienen del exterior.

El PIB ya nos dice algo sobre la evolución económica del país, pero nos dice poco sobre el porencial del bienestar económico de la población. Para conocer más sobre el potencial del bienestar económico observamos la evolución del Producto Intemo Bruto per cápita, PIBP, que nos dice en promedio cuánto le tocaría a cada residente del país si la producción se repartiera equitativamente entre todos los residentes. Esta cifra se obtiene de dividir el PIB entre la población tolal del país. Por eso hablo de "potencial del bienestar" y no de "bienestar" a secas. Insisto pues, que el PIBP sólo nos revela el potencial que el país tiene con respecto al bienestar material.

En la Gráfica 2 observamos que la evolución del PIBP es más quebrada que la del PIB, esto se debe a los cambios en la evolución del PIB y de la población. El PIBP no crece positivamente todos los años, a diferencia del PIB, pues en 
Gráfica 1

Producto interno bruto: 1950-1996

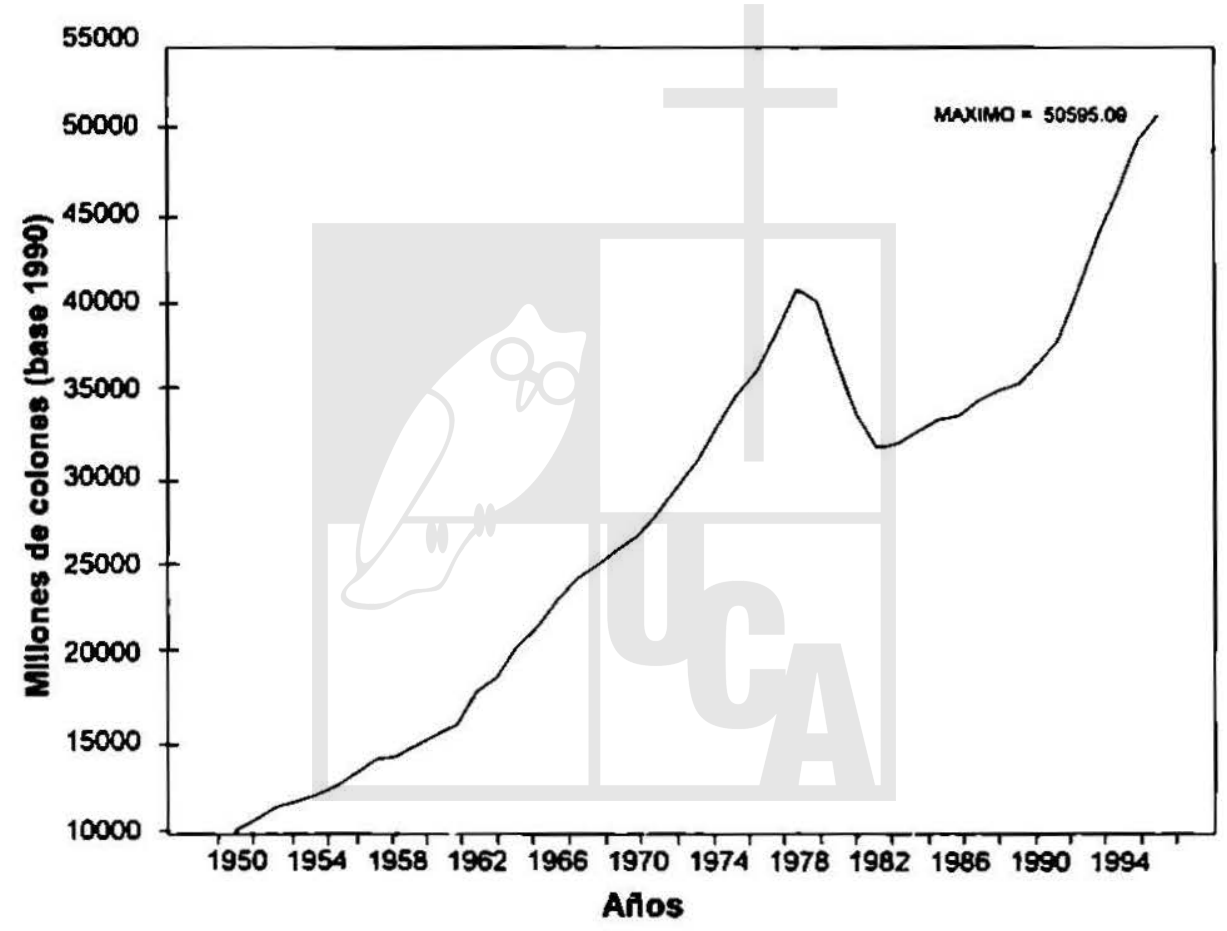


Gráfica 2

Producto interno bruto percápita: 1950-1996

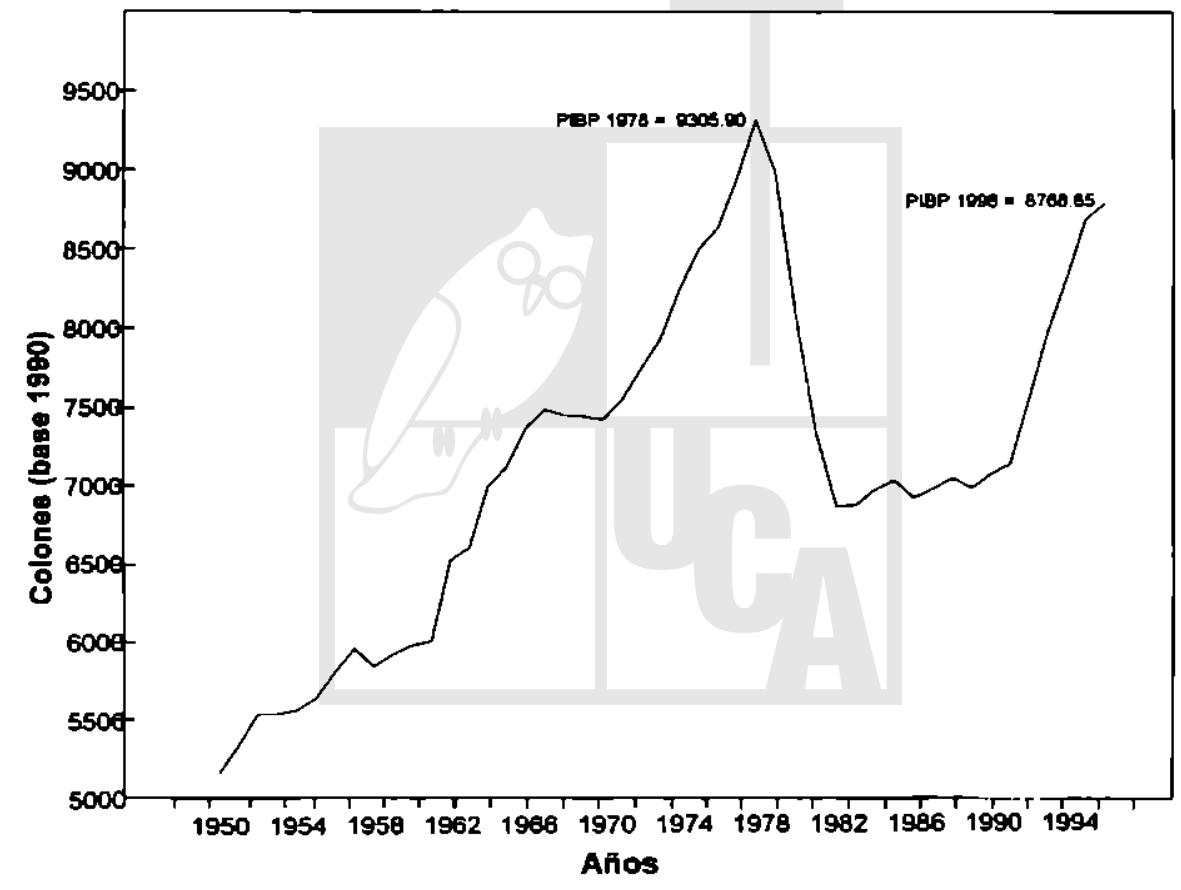

Digitalizado por Biblioteca "P. Florentino Idoate, S.J."

Universidad Centroamericana José Simeón Cañas 
algunos años el crecimiento de la producción total se ve acompañado por un crecimiento mayor en la población lo que hace que el PIBP disminuya, tal es el caso de los últimos años de la década de los sesenta con el agotamiento del Mercado Común Centroamericano. También notamos que en nuestro país, el nivel del PIBP más alto que alcanzamos fue en 1978. En 1996, el país aún no se había recuperado de la caída del PIBP de los primeros años de la década de los ochenta. El PIBP de 1996 era el equivalente al 94.2 por ciento del PIBP de 1978, es decir, que 18 años después de haber alcanzado el nivel del PIBP más alto en El Salvador, aún no nos hemos recuperado. La guerra civil le ha costado al país más de 18 años de pérdida en la mejoría del potencial de bienestar económico de la población.

\section{La tasa de crecimiento a largo plazo}

En esta sección se estiman las tasas de crecimiento del PIB y del PIBP a largo plazo. A diferencia de la sección anterior, en lugar de enfocarme en el crecimiento económico y la evolución del PIB y del PIBP en años determinados, nos concentramos en la evolución histórica promedio. Desde 1950 hasta 1996 voy a calcular cuáles han sido las tasas de crecimiento promedio para el PIB y el PIBP. Esta es una parte bastante técnica y el lector no interesado puede saltarse de una vez a la siguiente sección.

La manera más sencilla de obtener la tasa de crecimiento promedio de una variable es dibujar el logaritmo natural de la variable con respecto al tiempo y después, por medio de regresión simple, estimar la línea recta que mejor se ajusta a la tendencia temporal, y cuya pendiente representa la tasa de crecimiento promedio de la variable en cuestión. En los gráficos tres y cuatro las línea sólidas representan el logaritmo natural de los datos históricos del PIB y el PIBP, respectivamente. Al estimar una regresión simple de cada una de las variables con respecto a una constante y con respecto al tiempo, obtuve los siguientes resultados:

$$
\begin{aligned}
& \operatorname{In}(P I B)=9.37+0.032 \operatorname{In}(T I E M P O) \\
& \begin{array}{lll}
(203.88) *(19.29)^{*} & \mathrm{R}^{2}=0.88 & \text { D.W. }=0.06
\end{array} \\
& \ln (P I B P)=8.66+0.0079 \ln (T I E M P O) \\
& (267.83)^{*}(6.74) \quad \mathrm{R}^{2}=0.49 \quad \text { D.W. }=0.104
\end{aligned}
$$


$\ddot{\infty}$

Gráfica 3

PIB: 1950-1996

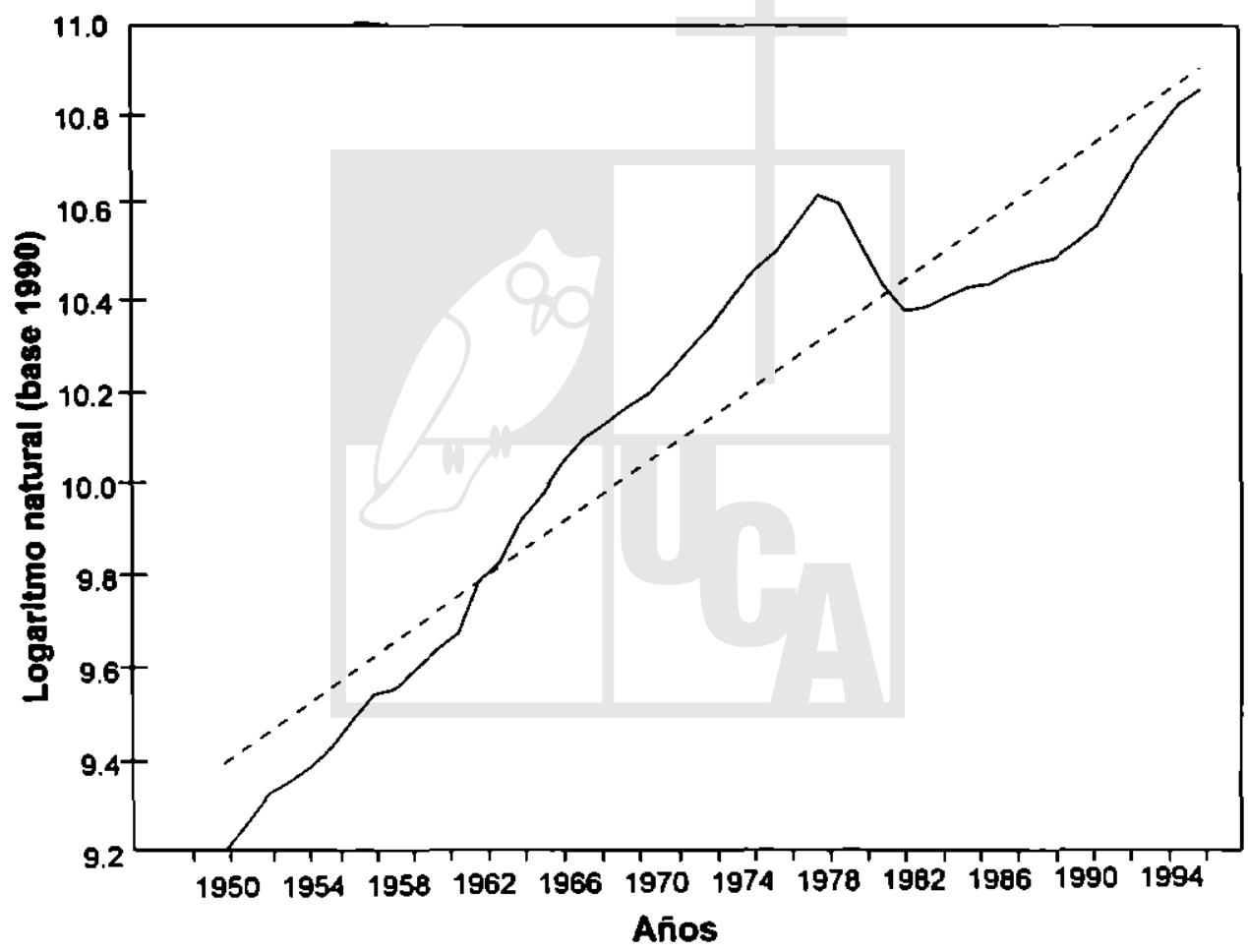

Digitalizado por Biblioteca "P. Florentino Idoate, S.J." Universidad Centroamericana José Simeón Cañas 
Gráfica 4

\section{PIB percápita 1950-1996}

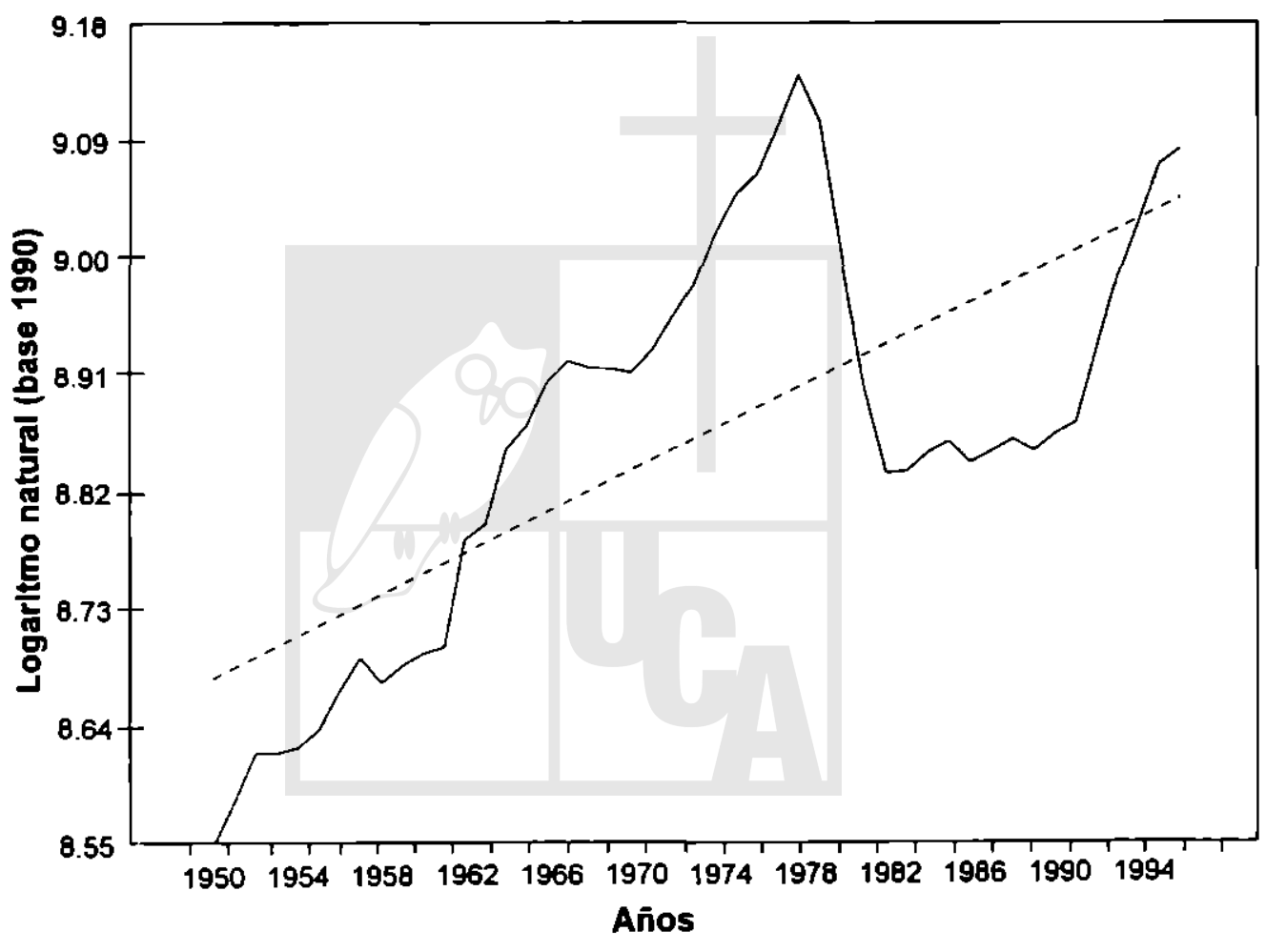


Donde ln es el operador de logaritmo natural, la variable tiempo es una variable que va de uno a 47 consecutivamente, $R^{2}$ es el coeficiente de determinación ajustado, y D.W. es el estadístico Durbin-Watson. Los números entre paréntesis son los estadísticos y $t$ el asterisco indica que los coeficientes estimados son estadísticamente significativos al uno por ciento ${ }^{2}$. Lo único que nos interesa en estas dos ecuaciones es el coeficiente que acompaña al logarítmo natural de la variable tíempo. Para el caso del PIB diríamos que la pendiente de la línea punteada en la Gráfica 3 es igual a 0.032 , lo que significa que la tasa de crecimiento promedio es de 3.2 por ciento. Para el caso del PIBP, en la Gráfica 4, dicho coeficiente es igual a 0.0079 , lo que significa que la tasa de crecimiento promedio del PIBP es de 0.79 por ciento.

En ambas ecuaciones el estadístico Durbin-Watson prueba que existe conrelación serial en el término de perturbación (no mostrado en la ecuación) lo que invalida las pruebas $t$ de significancia estadística. Cuando hay presencia de corelación serial hay varios métodos para tomarlos en cuenta a la hora de la estimación. Aplicando el mélodo de máxima verosimilitud de Beach-MacKinnon?, se obtiene que la tasa de crecimiento promedio del PIB es de 3.48 por ciento, $y$ la tasa de crecimiento del PIBP es de 1.07 por ciento, y ambas son estadisticamente significativas al uno por ciento.

Ya que lo que interesa es la estimación de las pendientes de las líneas punteadas en las Gráficas 3 y 4, utilizando los resultados anteriores podemos utilizar como aproximaciones los resultados de la estimación por el método BeachMacKinnon. En tal caso, asumiendo que existe una tendencia de crecimiento a largo plazo, una aproximación de las tasas de crecimiento de largo plazo del PIB y del PIBP son 3.48 por ciento y 1.07 por ciento, respectivamente. En la sección siguiente explico cómo interpretar estos resultados.

\section{La interpretación de las tasas de crecimiento}

Una vez obtenidas las tasas de crecimiento a largo plazo, lo que interesa conocer es su significado en palabras sencillas y asociadas a ideas más cotidianas, pues la cifra por sí sola no dice mucho. Para lograr esto recurriremos a la malemática. Suponiendo que el PIB y el PIBP crecen a las tasas de crecimiento constante estimadas en la sección anterior, su tendencia a largo plazo se puede representar por las siguientes ecuaciones:

$$
\begin{aligned}
& P I B_{1}=P I B_{o} \quad e^{0.0348,} \\
& P I B P_{1}=P I B P_{o} \quad e^{0.0107}
\end{aligned}
$$

Donde $\boldsymbol{e}$ es la base del logaritmo natural, los subíndices indican el momento en el tiempo, y 0 indica únicamente la fecha inicial, que en nuestro caso es 1950. 
Debido a que nos interesa conocer el crecimiento en el potencial de bienestar económico de la población, en lo que sigue haré mención únicamente al PIBP. Una pregunta interesante es, ¿cuánto tiempo tomaría duplicar el PIBP? Utilizando la ecuación cuatro podemos responder a esla pregunta. Si partimos del valor inicial sabemos que a una tasa de crecimiento constante en algún momento $T$, el $P I B P_{T}$ será el doble del valor inicial, por tanto $P I B P_{T}=2 P I B P_{0}$, luego utilizando la ecuación 4 , podemos sustituir al lado izquierdo la expresión correspondiente al período $T, y$ al lado derecho la expresión correspondiente al período 0 ,

$$
P I B P_{0} e^{0.0107 T}=2 P I B P_{0} \quad e^{(0.0107) 0}
$$

Los valores iniciales aparecen a ambos lados de la ecuación, por tanto, se pueden cancelar. En el lado derecho el exponente en el número $\boldsymbol{e}$ es cero, por tanto, $\boldsymbol{e}$ elevado a la cero se hace uno, y nos queda solamente,

$$
e^{0.0107 T}=2
$$

donde la única incógnita es $T$. Aplicando el logaritmo natural a ambos lados de esta ecuación, permite despejar el valor de $T$, el número de años que tomaría duplicar el PIBP,

$$
T=\frac{\operatorname{In}(2)}{0.0107}=64.78
$$

Lo que esto nos está indicando es que desde 1950 hasta 1996, el PIB per cápita en El Salvador a crecido a una tasa promedio aproximada de 1.07 por ciento, lo que significa que para duplicar el potencial de bienestar económico en la población nos tomaría aproximadamente 65 años. A manera de comparación, veamos el crecimiento del PIBP en otros países. Entre 1965 y 1989, el crecimiento del PIBP en Hong Kong fue de 6.3 por ciento, y les tomaría aproximadamente 11 años duplicar la producción percápita; en Corea del Sur, la tasa de crecimiento fue de 7, y les tomaría aproximadamente 10 años duplicar su producción; en Estados Unidos fue de 1.6 por ciento, y allí les tomaría aproximadamente 43 años duplicar la producción percápita4.

Puesto de otra forma, en todo el tiempo que nosotros nos tardaríamos en duplicar nuestro nivel de producción percápita, en Hong Kong se incrementaría a 60; en Corea del Sur casi 95 veces, y en Estados Unidos casi el triple. Aunque esto parece un juego de números, es algo más. Por un lado, nos expresa que nosotros hemos estado creciendo mucho más despacio que estos países y que de 
continuar así, las diferencias en los niveles de vida van a ser mucho más grandes. Por otro lado, nos expresa que nuestro país debe crecer más rápido, y así lo señalan otras voces, como Melhado (1997) y algunos ponentes de los seminarios de FUNDE y FUSADES, mencionados anteriormente. Incluso, se ha llegado a mencionar la cifra concreta del 7 por ciento en el crecimiento del PIB, lo que implica que si el crecimiento de la población es del orden del 2 por ciento, se está aspirando a un crecimiento del PIBP del 5 por ciento. Se quiere pues que dupliquemos nuestro ingreso aproximadamente cada 14 años, lo que eminentemente haría que en el término de la vida de una persona salvadoreña realmente se sentiría el mejoramiento del bienestar económico, en contraste con la tasa del 1.07 por ciento.

Aquí nos encontramos con un dilema. Nuestra tasa histórica de crecimiento del PIBP es aproximadamente del 1.07 por ciento, y se desea pasar a la categoría de países exitosos con un crecimiento del PIBP del 5 por ciento anual. El juego numérico del párrafo anterior nos muestra las grandes implicaciones que esto tiene para el potencial del bienestar económico del país. En lugar de duplicar nuestro potencial en 65 años, lo haríamos en 14. Una persona cuya vida laboral se extendiera unos 40 años vería elevar sus ingresos por menos del doble a lo largo de su vida laboral en el caso del 1.07 por ciento, mientras que vería aumentar sus ingresos por más de siete veces en el caso del 5 por ciento.

\section{Conclusión}

Volviendo a la preocupación inicial sobre la comprensión de la tasa de crecimiento económico podemos presentar este breve análisis. Durante casi medio siglo, la tasa de crecimiento económico en El Salvador, medida por la tasa de crecimiento del PIB percápita, ha sido de 1.07 por ciento. Esto significa que el ingreso promedio en El Salvador se logra duplicar cada 65 años. Si aspiramos pasar a la liga de los países exitosos y logramos una tasa de crecimiento del PIB percápita del 5 por ciento, el ingreso promedio se duplicaría en 14 años, y por supuesto la percepción en la población sería la de un mayor progreso económico. El problema radica en cómo hacer ese salto del 1.07 al 5 por ciento.

\section{Bibliografia}

Greene, William H. Econometric Analysis, Second Edition, Macmillan Publishing Company, 1993, pp. 791.

Melhado, Oscar. El Salvador, retos económicos de fin de siglo, UCA Editores, El Salvador, 1997.

World Bank, World Development Report 1991, Oxford University Press, 1991, pp. 290. 


\section{Apéndice I}

Tabla de datos

\begin{tabular}{|c|c|c|c|c|c|}
\hline Ano & Poblaclón & PIO & $x$ & PIBP & $\bar{Y}$ \\
\hline 1950 & 1940 & $1002 \theta$ & n.a. & 5170 & n.e. \\
\hline 1051 & 1988 & 10625 & $5.9 \%$ & $53 \overline{54}$ & $3.4 \%$ \\
\hline 1952 & 2039 & 11304 & $6.4 \%$ & 5543 & $3.7 \%$ \\
\hline 1053 & 2084 & 11604 & $2.7 \%$ & 5541 & $0.0 \%$ \\
\hline 1054 & 2152 & 11970 & $3.2 \%$ & 5565 & $0.4 \%$ \\
\hline 1955 & 2214 & 12483 & $4.2 \%$ & 5639 & $1.3 \%$ \\
\hline 1958 & 2278 & 13238 & $6.0 \%$ & 5811 & $3.0 \%$ \\
\hline 1857 & 2346 & 13983 & $5.6 \%$ & 5959 & $2.8 \%$ \\
\hline 1950 & 2418 & 14128 & $1.0 \%$ & 5844 & $-1.0 \%$ \\
\hline 1859 & 2492 & 14759 & $4.5 \%$ & 5922 & $1.3 \%$ \\
\hline 1960 & 2570 & 15358 & $4.1 \%$ & 5978 & $0.9 \%$ \\
\hline 1981 & 2840 & 15907 & $3.6 \%$ & 6005 & $0.5 \%$ \\
\hline 1862 & 2730 & 17790 & $11.0 \%$ & 6520 & $8.6 \%$ \\
\hline 1993 & 2814 & 10565 & $4.3 \%$ & 6597 & $1.2 \%$ \\
\hline 1964 & 2906 & 20302 & $0.4 \%$ & 6986 & $5.9 \%$ \\
\hline 1965 & 3008 & 21388 & $5.3 \%$ & 7115 & $1.8 \%$ \\
\hline 1066 & 3115 & 22920 & $7.2 \%$ & 7350 & $3.4 \%$ \\
\hline 1087 & 3232 & 24168 & $5.4 \%$ & 7477 & $1.6 \%$ \\
\hline 1968 & 3354 & 24948 & $3.2 \%$ & 7438 & $-0.5 \%$ \\
\hline 1569 & 3474 & 25817 & $3.5 \%$ & 7431 & $-0.1 \%$ \\
\hline 1070 & 3588 & 26586 & $3.0 \%$ & 7410 & $-0.3 \%$ \\
\hline 1071 & 3696 & 27866 & $4.8 \%$ & 7540 & $1.8 \%$ \\
\hline 1972 & 3798 & 29388 & $5.5 \%$ & 7738 & $2.8 \%$ \\
\hline 1973 & 3894 & 30876 & $5.1 \%$ & 7928 & $2.5 \%$ \\
\hline 1974 & 3989 & 32859 & 6.4\% & 8237 & $3.0 \%$ \\
\hline 1975 & 4085 & 34687 & $5.6 \%$ & 8491 & $3.1 \%$ \\
\hline 1976 & 4181 & 36004 & $4.0 \%$ & 8626 & $1.6 \%$ \\
\hline 1977 & $427 \theta$ & 38252 & $6.1 \%$ & $69 \overline{39}$ & $3.6 \%$ \\
\hline 1978 & 4374 & 40704 & $6.4 \%$ & 9306 & $4.1 \%$ \\
\hline 1979 & 4457 & 40004 & $-1.7 \%$ & 6976 & $-3.5 \%$ \\
\hline 1980 & 4525 & 36535 & $-8.7 \%$ & 8074 & $-10.0 \%$ \\
\hline 1981 & 4570 & 33508 & $-0.3 \%$ & 7318 & $-9.4 \%$ \\
\hline 1082 & 4817 & 31630 & $-5.6 \%$ & 6851 & $-0.4 \%$ \\
\hline 1083 & 4648 & 31862 & $0.8 \%$ & 6859 & $0.1 \%$ \\
\hline 1884 & 4686 & 32606 & $2.3 \%$ & 6958 & $1.4 \%$ \\
\hline 1085 & 4739 & 33250 & $2.0 \%$ & 7016 & $0.8 \%$ \\
\hline 1980 & 4840 & 33460 & $0.6 \%$ & 6905 & $-1.6 \%$ \\
\hline 1987 & 4934 & 34360 & $2.7 \%$ & 6964 & $0.9 \%$ \\
\hline 1888 & 4969 & 34919 & $1.0 \%$ & 7027 & $0.9 \%$ \\
\hline 1889 & 5097 & 35287 & $1.1 \%$ & 6984 & $-0.8 \%$ \\
\hline 1900 & 5172 & 38467 & $3.4 \%$ & 7055 & $1.3 \%$ \\
\hline 1991 & 5311 & 37791 & $3.9 \%$ & $711 \theta$ & $0.9 \%$ \\
\hline 1992 & 5388 & 40843 & $7.5 \%$ & 7529 & $5.8 \%$ \\
\hline 1993 & 5487 & 43643 & $7.4 \%$ & 7954 & $5.6 \%$ \\
\hline 1994 & 5577 & 46252 & $6.0 \%$ & 8294 & $4.3 \%$ \\
\hline 1995 & 5668 & 49144 & 6.3\% & 8670 & $4.5 \%$ \\
\hline 1900 & 5770 & 50595 & $3.0 \%$ & 8789 & $1.1 \%$ \\
\hline 1897 & 5874 & 52619 & $4.0 \%$ & 8958 & $2.2 \%$ \\
\hline
\end{tabular}

Fuente: Elaboración propia con base en los datos de la Revisla Trimestral del Banco Central de Reserva, varios años; Memoria de Labores del Banco Central de Reserva, 1984. Anuario de las Estadísticas Financicras Internacionales del Fondo Monetario Internacional, varios años. 


\section{Notas}

1. Entre otros, ver el nucvo libro de Oscar Melhado (1997). Tales preocupaciones también se hicicron presentes en dos seminarios a los que asistí durante la segunda milad de 1997: "Los determinantes del ahorto y la inversión en la cconomía salvadoreña. 1970-1995", cuyo ponente fuc Francisco Lazo Marin, palrocinado por FUNDE, y el otro seminario titulado "Estabilización y perspectivas de crecimiento en El Salvador", cuyos ponentes fueron Vittorio Corbo, Felipe Larraín y Guillermo Calvo, patrocinado por FUSADES.

2. La hipbtcsis nula es que el coeficiente es igual a ccro. Un t estadistico bastantc alto lleva a concluir que el coeficiente es significativamente diferente de cero.

3. Ver Greene (1993), p. 432-433.

4. Esios datos provienen de World Bank (1991), Tabla 1, pág. 204. 